\title{
TRYPANOSOMA LEWISI ET SPLÉNECTOMIE
}

\section{Par J. SCHWETZ}

On sait que la splénectomie réveille parfois de nombreuses infections parasitaires latentes, chez les animaux prémunis contre ces affections : piroplasmose, anaplasmose, etc. (E. Brumpt, 1929).

En ce qui concerne l'influence de la splénectomie sur l'infection par $T$. lewisi, nous possédons deux études; une de Regendanz et Kikuth (1927), et une autre de Bruynoghe et Vassiliadis (1929).

Regendanz et Kikuth ont infecté avec plusieurs souches de $T$. lewvisi deux séries de rats normaux et splénectomisés et ont constaté une différence dans le cours de l'infection entre les deux groupes de rats. Cette différence était pour ainsi dire quantitative et qualitative. La durée de la période de multiplication était de 4 jours en moyenne chez les rats splénectomisés, au lieu de un à deux jours chez les rats normaux. Ensuite, le nombre des trypanosomes était plus grand chez les rats splénectomisés que chez les rats normaux. Enfin, quelques-uns des rats splénectomisés sont morts de leur trypanosomose. Par contre, Brumpt n'a pas remarqué une augmentation marquée du nombre des $T$. lewisi chez deux rats infectés, depuis environ six mois, pendant les semaines qui ont suivi l'opération.

Bruynoghe et Vassiliadis ont essayé de transmettre $T$. lewisi à des rongeurs qui normalement n'en sont pas infectés, en faisant simultanément ces essais sur des animaux normaux et splénectomisés. Le résultat chez la souris naine (chez les autres rongeurs le résultat était toujours négatif) fut le suivant :

$$
\begin{aligned}
& \text { Non splénectomisées......... pas d'infection (dans } 4 \text { souris) } \\
& \text { Splénectomisées........... }
\end{aligned}
$$

Ces expériences démontrent que la splénectomie peut rendre certains animaux réceptifs pour l'infection à $T$. lewisi, à l'égard de laquelle ils étaient, avant l'enlèvement de la rate, totalement réfractaires.

En ce qui concerne le rat, hôte habituel et normal, pour ainsi dire, de T. Lewisi, Bruynoghe et Vassiliadis écrivent ce qui suit :

"L'évolution de la trypanosamiase des rats fut manifestement " aggravée par la splénectomie ; la plupart des rats (Mus rattus,

Annales de Parasitologie, T. IX, $\mathrm{N}^{\circ} 1$ - $1^{\text {er }}$ janvier 1931 , p. 10-14. 
"Mus decumanus et rats blancs) ont succombé au cours de leur " infection; et les quelques autres ne sont arrivés à la guérison " qu'après une infection très grave, accompagnée d'altérations " sanguines évidentes (anisocytose, poikilocytose, de nombreux " globules rouges nucléés, etc.), Dans nos expériences, l'aggrava* tion ne pouvait être attribuée à une bartonellose concomittante, « étant donné que les splénectomisés avaient été guéris de cette " infection par un traitement arsenical. »

Bruynoghe et Vassiliadis ne disent pas s'il s'agissait de rats normalement infectés avant la splénectomie ou artificiellement infectés après l'opération. Quoi qu'il en soit, ces auteurs parlent de symptômes cytologiques et cliniques provoqués par T. lewisi, tandis que Regendanz et Kikuth parlent de l'évolution de ce dernier parasite lui-même.

Les conclusions ne sont en somme pas bien nettes. On se demande aussi quel est le tableau cytologique des rats splénectomisés, en l'absence de $T$. lewisi et de Bartonella. Personnellement nous avons constaté une très forte anémie chez des rats splénectomisés, même en l'absence de ces deux parasites (1930).

On remarquera que dans les expériences de Regendanz et Kikuth, il s'agit d'une infection expérimentale chez des rats splénectomisés ayant en outre reçu une injection de salvarsan pour éviter la bartonellose.

Mais que se passe-t-il chez les rats naturellement infectés par $T$. lewisi et splénectomisés ? L'opération aggrave-t-elle l'infection ou non ? Nous avons déjà cité la constatation de Brumpt. Dans leur note très intéressante sur l'anémie à Bartonella des rats splénectomisés, Bonnin et Jonchères se bornent à dire ce qui suit sur le sujet qui nous intéresse : " les trypanosomes ( $T$. lewisi, 95 p. 100 des rats de Bordeaux) ont augmenté passagèrement de nombre du $3^{\circ}$ au $7^{\circ}$ jour $\gg$.

Mais surtout que se passe-t-il chez des rats indemnes de trypanosomes et splénectomisés ?

L'ablation de la rate fait-elle sortir, apparaître, les trypanosomes dans le sang périphérique ?

Nous tâcherons d'apporter à ces questions une modeste contribution par nos observations.

Comme nous l'avons déjà signalé dans une note précédente (1929), les rats de Stanleyville (Rattus rattus frugivorus) sont naturellement infectés par $T$. lewisi dans une très forte proportion. Sur 57 rats, 'notamment, nous en avions trouvé 16 infectés, soit 28 p. 100. Citons ici le passage suivant de la note précédente :

«Quelquefois assez rares, les trypanosomes étaient en général 
bien nombreux dans les frottis. Parfois ils y grouillaient, pour ainsi dire. Il s'agissait toujours de $T$. lewisi typique habituel, de formes adultes. Nous n'avons jamais trouvé de formes d'évolution. Et si la proportion de trypanosés était très grande parmi les rats adultes, nous n'avons, par contre, jamais trouvé de trypanosomes chez les très jeunes rats (1). »

Depuis la rédaction de la note précédente, en juillet 1923 , nous avons eu l'occasion d'examiner d'autres rats avec plus ou moins le même résultat, sauf que chez un seul nous avons enfin trouvé des formes de division et même des rosaces, en même temps d'ailleurs que des trypanosomes adultes.

Quant aux rats splénectomisés, leur examen préalable, c'est-àdire avant l'opération, nous a donné le résultat suivant (et disons, en passant, que nous n'avons splénectomisé que des rats bien solides, c'est-à-dire adultes et même âgés) :

I. - 14 indemnes de trypanosomes.

II. -6 avec $T$. lewisi.

Le tableau de l'infection trypanosomique chez les 6 rats de la deuxième catégorie était le suivant :

Rat 1. - Rares trypanosomes avant l'opération, et rares après l'opération ; à partir du $25^{\circ}$ jour après la splénectomie on ne trouve plus de trypanosomes, mais le $40^{\circ}$ jour ils réapparaissent en assez grand nombre avec des formes de multiplication. Le rat meurt le lendemain.

Rat 2. - Rares trypanosomes aussi bien avant la splénectomie que durant 30 jours après l'opération.

Rat 3. - Nombreux trypanosomes aussi bien avant l'opération que durąnt les 40 jours de survie après la splénectomie.

Rat 4. - Nombreux trypanosomes à l'examen préalable et durant les 40 jours de survie post-splénectomique. Vu, le $2^{\circ}$, le $4^{\circ}$ et le $33^{\circ}$ jours après l'opération, quelques trypanosomes en division et même des rosaces.

Rat 5. - Nombreux trypanosomes à l'examen préalable et durant les 70 jours de survie post-splénectomique. Vu, le $21^{\circ}$ jour après l'opération, quelques formes de multiplication.

Rat 6. - Très rares trypanosomes, aussi bien à l'examen préalable que durant les 38 jours de survie post-opératoire.

(1) Dans l'analyse de ma note, faite par M. Mesnil dans le Bulletin de l'Institut Pasteur (1930), il s'est glissé une erreur. Il y est dit : « Les très jeunes rats ont montré des lewisi ". Or, comme on vient de le voir par la citation, c'est le contraire : c'est justement chez les très jeunes rats que nous n'avons jamais trouvé de trypanosomes. 
Au point de vue quantitatif, la splénectomie n'a donc eu aucune influence chez les six rats en question. Quand les trypanosomes étaient rares avant la splénectomie, ils restaient rares après elle, et vice-versa. Mais chez trois rats sur six nous avons vu apparaitre, dans la période post-splénectomique, des formes de multiplication. Etait-ce la conséquence de la splénectomie ? C'est possible, mais pas certain. En effet, si chez le rat 4 des formes de division apparurent dès le $2^{\circ}$ jour après l'opération, elles ne se montrèrent que le $21^{\circ}$ jour chez le rat 5 et même le $40^{\circ}$ jour, in extremis, chez le rat 1 .

Les 14 rats trouvés indemnes de trypanosomes à l'examen préalable, avant la splénectomie, doivent être divisés en deux lots inégaux :

1. Chez le premier lot de 12 rats, nous n'avons pas trouvé de trypanosomes durant l'observation post-opératoire, soit jusqu'à la mort, soit jusqu'à la disparition de l'animal. La durée de l'observation était respectivement de : $17,24,65,15,95,16,35,58,60,52$, 36 et 15 jours.

2. Quant au deuxième lot de 2 rats sans trypanosomes avant la splénectomie, nous avons constaté ceci :

A. Rat 13. - Trouvé quelques rares trypanosomes ordinaires le $7^{\circ}$ jour après la splénectomie. Le $10^{\circ}$ jour très nombreux trypanosomes en division et rosaces. De même les $11^{\circ}$ et $13^{\circ}$ jours. Le $14^{\circ}$ jour on ne voit que des trypanosomes ordinaires qui persistent jusqu'au $50^{\circ}$ jour, date de la mort de l'animal. La durée de la multiplication a done été de 3 ou de 4 jours. Mais il ne faut pas oublier que nos rats splénectomisés avaient des puces et pouvaient s'infecter aussi bien après la splénectomie qu'avant l'opération.

B. Rat 14. - Pas vu de trypanosomes jusqu'au $34^{\circ}$ jour après l'opération. Mais, ce jour-là, nous trouvons quelques trypanosomes, d'ailleurs uniquement adultes. Par une malheureuse coïncidence, le rat disparaît (avec plusieurs autres) le lendemain.

\section{RÉSUMÉ}

Chez 12 rats sur 14, la splénectomie n'a pas fait apparaître de trypanosomes. Quant aux deux autres, l'influence de la splénectomie reste bien douteuse, malgré l'apparition de trypanosomes dans la période post-opératoire. Chez le rat 14 , les trypanosomes n'ont apparu que le $34^{\mathrm{e}}$ jour après l'opération. En somme, ce n'est que dans le rat 13 qu'on peut attribuer l'apparition des trypanosomes à la splénectomie. Il s'agissait alors d'un réveil d'une infection 
latente antérieure. Mais il est aussi possible que ce soit une simple coïncidence, c'est-à-dire une infection indépendante de la splénectomie et produite par les puces de ce rat après l'opération.

En somme, pour élucider définitivement la question, il faudrait observer simultanément, et pendant assez longtemps, deux groupes de rats, trypanosomés et sans trypanosomes, chacun de ces deux groupes étant divisé en deux lots : splénectomisés et non splénectomisés. Mais, dès à présent, on peut dire, en se basant sur les observations de nos 20 rats, que la splénectomie ne paraît pas exercer une influence notable sur l'évolution de $T$. lewisi. Si nous avons trouvé plus souvent des formes de multiplication chez les rats splénectomisés que chez les non splénectomisés, c'est, très probablement, parce que les premiers ont été examinés à plusieurs reprises pendant longtemps, tandis que chez les seconds, on n'avait fait qu'un simple examen. Il s'agit, en d'autres termes, de quelques trouvailles occasionnelles.

En résumé, nous dirons que l'influence de la splénectomie sur l'évolution de $T$. lewisi reste encore à démontrer.

\section{BIBLIOGRAPHIE}

Bonnin (H.) et Jonchères (K.). - L'anémie à Bartonella des rats splénectomisés. C. R. Soc. biol., CI, 1929, p. 681.

Brumpt (E.). - Splénectomie et infections parasitaires. Arch. d'anatomie microscopique, XXV, 1929, p. 49-55.

Bruynoghe (R.) et Vassiliadis. - La splénectomie dans l'infection du T. lewisi. Ann. Soc. Belge de médecine tropicale, IX, 1929, p. 191-195.

Regendanz (P.) und Kinuth (W.). - Ueber die Bedeutung der Milz für die Bildung der vermehrungshindernden Reaktionsproduktes und dessen Wirkung auf den Infektionsverlauf der Ratten-Trypanosomiasis (Tryp. lewisi). Centralblatt für Bakter., CIII, 1927, p. 271-279.

Schwetz (J.) et CaBu (F.), - Note préliminaire sur les Grahamella-Bartonella des rats splénectomisés de Stanleyville (Congo Belge). Bull. Soc. de pathologie exotique, XXIII, 1930 p.464-473, pl. V-VI.

Schwetz (J.) et Mlle Geerinck. - Sur quelques parasites sanguicoles des rongeurs de Stanleyville (Congo Belge). Bull. Soc. de pathologie exotique, XXII, 1929, p. 657-661. Bull. de l'Institut Pasteur, XXVIII, 1930, p. 742. 